\title{
Efficacy of omega-3 fatty acid supplementation on cardiovascular risk factors in patients with polycystic ovary syndrome: a systematic review and meta-analysis
}

\author{
Yidan $\mathrm{Xia}^{1,2 \#}$, Yan Wang ${ }^{1,2 \#}$, Manman $\mathrm{Cui}^{1,2}$, Dan $\mathrm{Su}^{1,2}$ \\ ${ }^{1}$ Department of Gynecology and Obstetrics, Sichuan Provincial People's Hospital, University of Electronic Science and Technology of China, \\ Chengdu, China; ${ }^{2}$ Chinese Academy of Sciences Sichuan Translational Medicine Research Hospital, Chengdu, China \\ Contributions: (I) Conception and design: D Su, M Cui; (II) Administrative support: Y Wang; (III) Provision of study materials or patients: Y Xia, Y \\ Wang; (IV) Collection and assembly of data: Y Xia, Y Wang; (V) Data analysis and interpretation: Y Xia, D Su; (VI) Manuscript writing: All authors; \\ (VII) Final approval of manuscript: All authors. \\ "These authors contributed equally to this work as co-first authors. \\ Correspondence to: Manman Cui; Dan Su. Department of Gynecology and Obstetrics, Sichuan Provincial People’s Hospital,University of Electronic \\ Science and Technology of China, 32 West Second Section, First Ring Road, Chengdu 610072, China; Chinese Academy of Sciences Sichuan \\ Translational Medicine Research Hospital, Chengdu, China. Email: 121047742@qq.com; sudansichuan@163.com.
}

Background: There is a close relationship between cardiovascular risk factors and polycystic ovary syndrome (PCOS), and omega-3 fatty acids may have a key role in improving cardiovascular risk factors. We conducted the current systematic review and meta-analysis to evaluate the effect of omega-3 fatty acid supplementation on cardiovascular risk factors in patients with PCOS.

Methods: We searched 4 databases including PubMed (MEDLINE), Cochrane Library, Embase, and Web of Science from inception to February 2021. We included randomized controlled trials (RCTs) that reported the effects of omega-3 fatty acid treatment for PCOS. According to the Cochrane system evaluation guide manual, 2 researchers independently assessed the methodological quality of the included studies. We pooled results using either a fixed effect model or random effect model.

Results: We identified 314 articles, of which 10 met the criteria for inclusion, involving 778 participants. The pooled results suggested an association between the supplementation of omega- 3 fatty acids and a reduction in serum insulin $[-2.58 \mathrm{pmol} / \mathrm{L}, 95 \%$ confidence interval $(\mathrm{CI}):-3.34$ to $-1.82 \mathrm{pmol} / \mathrm{L}, \mathrm{P}<0.00001$, $\left.\mathrm{I}^{2}=0\right]$, homeostatic model assessment of insulin resistance (HOMA-IR) (-0.57, 95\% CI: -0.75 to $-0.40 \mathrm{~L}$, $\left.\mathrm{P}<0.00001, \mathrm{I}^{2}=2 \%\right)$, serum total cholesterol (TC) $(-6.87 \mathrm{mg} / \mathrm{dL}, 95 \%$ CI: -10.28 to $-3.47 \mathrm{mg} / \mathrm{dL}, \mathrm{P}<0.0001$, $\mathrm{I}^{2}=95 \%$ ), serum triglyceride $\left(-4.03 \mathrm{mg} / \mathrm{dL}, 95 \% \mathrm{CI}:-5.53\right.$ to $\left.-2.52 \mathrm{mg} / \mathrm{dL}, \mathrm{P}<0.00001, \mathrm{I}^{2}=97 \%\right)$, serum lowdensity lipoprotein cholesterol (LDL-C) (-6.64 mg/dL, 95\% CI: -11.58 to $-1.70 \mathrm{mg} / \mathrm{dL}, \mathrm{P}=0.008, \mathrm{I}^{2}=99 \%$ ), serum very low-density lipoprotein cholesterol (VLDL-C) $(-3.29 \mathrm{mg} / \mathrm{L}, 95 \% \mathrm{CI}:-6.54$ to $-0.05 \mathrm{mg} / \mathrm{L}$, $\left.\mathrm{P}=0.05, \mathrm{I} 2=7^{2} \%\right)$, serum high-sensitivity C-reactive protein (hs-CRP) $(-8.97 \mathrm{mg} / \mathrm{dL}, 95 \%$ CI: -17.66 to $0.28 \mathrm{mg} / \mathrm{dL}, \mathrm{P}=0.04, \mathrm{I}^{2}=99 \%$ ), an improvement in serum high-density lipoprotein cholesterol (HDL-C) (2.94 mg/dL, $95 \% \mathrm{CI}: 0.63$ to $\left.5.26 \mathrm{mg} / \mathrm{dL}, \mathrm{P}=0.01, \mathrm{I}^{2}=87 \%\right)$, but no effect on serum glucose $(-0.76 \mathrm{mg} / \mathrm{dL}$, $95 \%$ CI: -1.71 to $0.19 \mathrm{mg} / \mathrm{dL}, \mathrm{P}=0.12, \mathrm{I}^{2}=73 \%$ ) was found.

Discussion: The current meta-analysis demonstrated that omega-3 fatty acid supplementation for women with PCOS resulted in a statistical improvement in insulin, HOMA-IR, TC, triglyceride, LDL-C, VLDL-C, and HDL-C, but did not affect serum glucose. The limitation of this paper is due to the lack of included research literature.

Keywords: Omega-3 fatty acids; meta-analysis; polycystic ovary syndrome (PCOS); cardiovascular risk factors

Submitted Apr 07, 2021. Accepted for publication Jun 15, 2021.

doi: 10.21037/apm-21-1050

View this article at: https://dx.doi.org/10.21037/apm-21-1050 


\section{Introduction}

Polycystic ovary syndrome (PCOS), with a prevalence of $6-10 \%$, is a common endocrine disorder in women (1). Aside from the reproductive consequences, PCOS is also linked to a higher risk of metabolic and cardiometabolic diseases (CVD), which have an important effects on long-term health (2). Besides, the major modifiable risk factors, including hyperglycemia, hyperinsulinemia, and dyslipidemia, frequently co-occur with PCOS. According to the National Cholesterol Education Program (NCEP) guidelines, up to $70 \%$ of PCOS women exhibit dyslipidemia and approximately $50-70 \%$ of lean and obese women with PCOS also have intrinsic insulin resistance (IR) (3).

So far, there is no approved pharmacologic therapy for PCOS, most of the drugs, such as oral contraceptives, antiandrogens, insulin sensitizers, and aromatase inhibitors, are used to treat the symptoms of PCOS and are used in an off label manner (4). Up to date, lifestyle modifications and weight loss are still remain the significant therapies of PCOS treatments. Recently, nutrition intervention has become a recommended treatment for PCOS women. Among the nutritional components, dietary fat is currently considered a biological regulator that affects all aspects of metabolism. Omega-3 fatty acids may be the most effective fatty acid regulators in metabolic function, which also have beneficial effects on the risk of cardiovascular disease $(5,6)$. Previous studies have indicated that therapy with omega-3 fatty acids positively impact cardiometabolic and lipid profiles (7) and induced IR (8). Meanwhile, animal studies have also found that omega-3 fatty acid supplementation can improve IR via activating peroxisome proliferatoractivated receptor alpha (PPARa), decreasing inflammation and fat accumulation in insulin sensitive tissues (9). However, the results concerning the effect of omega- 3 fatty acid supplementation on glucolipid metabolism in PCOS patients have been controversial. A study by Akinkuolie et al. (10) indicated that omega-3 fatty acids have no benefit on IR in patients with PCOS.

Considering the current controversy, and based on the available randomized controlled trials (RCTs), we designed this systematic review and meta-analysis to explore the effect of omega-3 fatty acid supplementation on the glucolipid metabolism of women with PCOS, which may help to inform clinicians and patients on the use of omega-3 fatty acids for PCOS with cardiovascular risks. We present the following article in accordance with the PRISMA reporting checklist (available at https://dx.doi.org/10.21037/ apm-21-1050) (11).

\section{Methods}

\section{Search strategy}

A total of 2 authors respectively searched 4 databases including Pubmed (Medline), the Cochrane Library, Web of science, and EMBASE from inception until 1 February 2021. Based on the PICOS principle, the search was conducted with the following keywords: ("omega-3 fatty acids acid" OR "omega-3" OR "docosahexaenoic acid" OR "PUFA" OR "EPA" OR "DHA" OR “ $\omega-3$ " OR "eicosapentaenoic") AND ("polycystic ovary syndrome" OR "PCOS"). If the data was incomplete, we attempted to contact the relevant author. In addition, the Clinical Register (clinicaltrials.gov) was searched if necessary. The reference lists of reviewed articles and reviews were manually searched for further relevant studies, with no limitation on language.

\section{Selection criteria}

According to the following principles, 2 authors respectively reviewed all literatures. Any disagreements will be resolved by consulting with the third reviewers

(I) The inclusion criteria were as follows: (i) population: patients with a definitive diagnosis of PCOS, aged $>18$ years; (ii) intervention measure: omega- 3 fatty acids at any dose and route; (iii) control group: placebo; (iv) outcomes: glycometabolism [serum glucose, serum insulin, homeostatic model assessment-insulin resistance (HOMA-IR)] serum lipid profile [total cholesterol (TC), triglyceride, low-density lipoprotein cholesterol (LDL-C), very low-density lipoprotein cholesterol (VLDL-C), and high-density lipoprotein cholesterol (HDL-C)]; and (v) study type: RCTs.

(II) The exclusion criteria were as follows: (i) not an RCT; (ii) duplicate publications; (iii) letters to the editor, conference papers, and articles only available in abstract form; and (iv) no assessment of primary data.

\section{Data extraction}

Based upon the criteria of inclusion and exclusion, all identified data were reviewed by 2 reviewers independently 


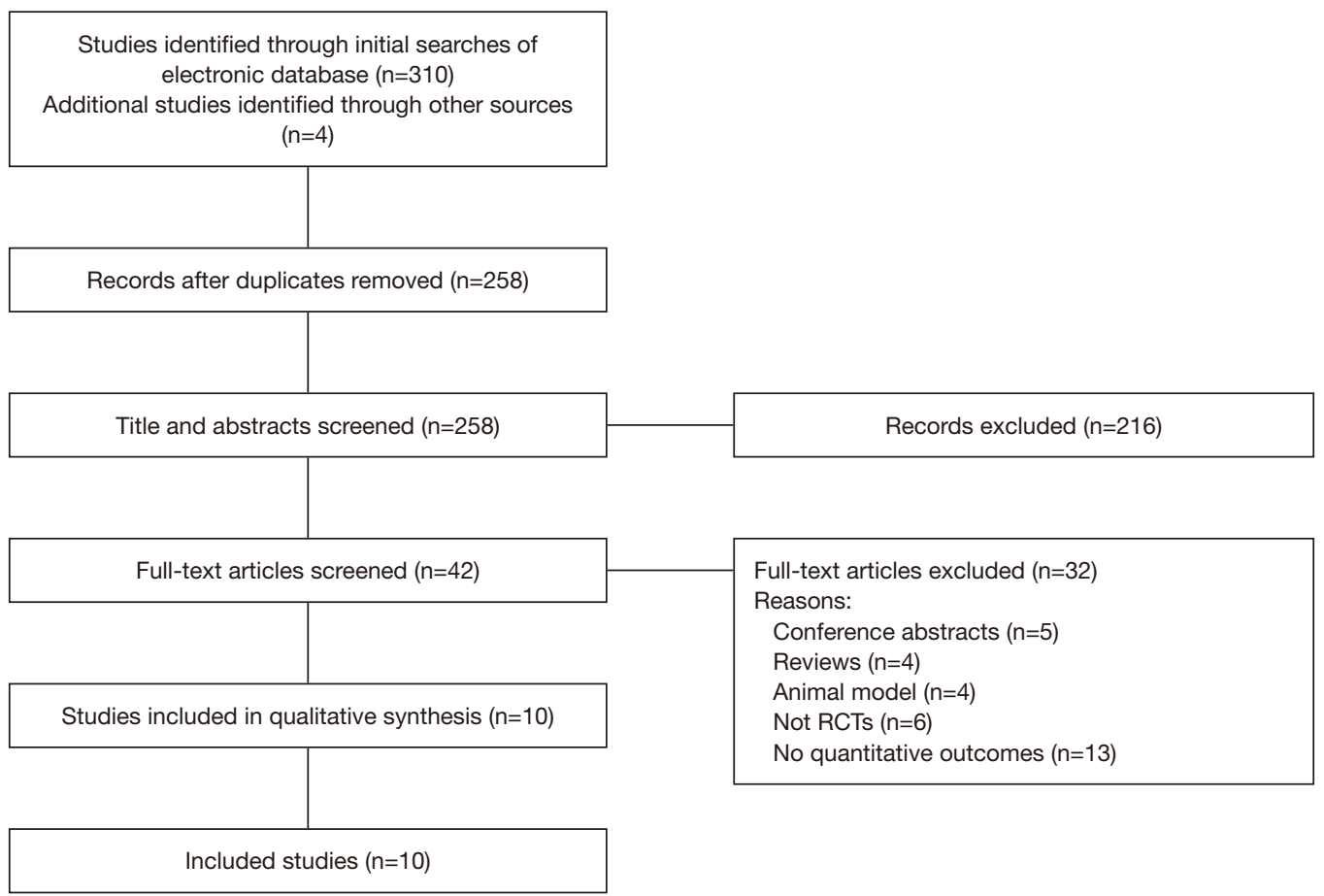

Figure 1 Flow diagram of study selection. RCT, randomized controlled trial.

and duplicate literature was removed. We collected the following information: first author, published year, sample size, study design, intervention characteristics (such as dosage, treatment duration, and so on), control characteristics, and outcome data (including serum glucose, serum insulin, HOMA-IR, TC, triglyceride, LDL-C, VLDL-C, and HDL-C). If the original data was not directly provided in the text or table, the figures in the study were referred to. If the relevant details were not reported in the study, we attempted to contact the authors for further information.

\section{Quality assessment}

According to the Cochrane system evaluation guide manual (version 5.1.0, https;//handbook-5-1.cochrane.org), 2 researchers independently assessed the methodological quality of the included studies (12). Any disagreements were resolved by consensus after discussion or referral to a third reviewer as appropriate. The assessment was divided into high, low, or unclear bias risks according to the following areas: random sequence generation, allocation concealment, degree of blindness (participant and outcome assessment), incomplete outcome data, selective outcome reporting, and other biases.

\section{Statistical analysis}

The data were analyzed by the use of RevMan 5.3 software (Review Manager, Copenhagen: The Nordic Cochrane Center, The Cochrane Collaboration, 2014). All results were described and analyzed with mean \pm standard deviation (SD). Weighted mean differences (WMD) and $95 \%$ confidence interval (CI) were used to measure all results. If the original data had not been reported, we used the established method to estimate the unreported mean \pm SD through other information provided in the publication (such as CIs or median and quartile interval) (13). The heterogeneity was analyzed by the Q test and $\mathrm{I}^{2}$ test. When the included studies had large heterogeneity $(\mathrm{P}<0.10$ or $\mathrm{I}^{2}>50 \%$ ), the random-effects model was used to collect the estimates of WMD in the study, otherwise, the fixed-effects model was be used. Publication bias was judged via the use of a funnel plot (14).

\section{Results}

As shown in Figure 1, 314 citations were identified. A total 
of 42 studies were selected for full-text review after the screening of the titles and abstracts. Of these 42 citations, 32 studies did not meet the inclusion criteria and were thus excluded. Finally, 10 studies (15-24) were enrolled in this systematic review and meta-analysis.

The characteristics of the 10 studies included in this study (Table 1) were summarized, involving 778 subjects, 385 of which were randomly divided into an omega- 3 fatty acids group and the rest as a control group. The sample size ranged from 34 to 195 . The dosage of omega-3 fatty acids was $1,000-4,000 \mathrm{mg} / \mathrm{d}$ for $6-24$ weeks.

\section{Quality assessment}

The quality evaluation results of RCTs included in our study are shown in Figure 2. A total of 7 trials (15-21) performed randomization with the use of a computergenerated random list, and 3 RCTs (22-24) did not provide detailed information of the randomization method. With regard to allocation, 3 trials $(15,19,20)$ were classified as low risk and allocation concealment was used appropriately, and the others (16-18,21-24) were classified as unclear risk. A total of 8 of the RCTs $(15,16,18-22,24)$ included in our study were characterized by a low risk of blinding of participants and personnel and outcome assessment, and 2 trials $(17,23)$ were categorized as unclear. As for the blinding of outcome assessment and outcome data, all RCTs (15-24) were classified as low risk. Selective reporting was assessed, and only 1 trial (15) was classified as low risk; the remaining 9 (16-24) were classified as other biases unclear.

\section{Pooled analysis}

Effect of omega-3 fatty acid supplementation on serum glucose, serum insulin, and HOMA-IR

Meta-analysis of 6 RCTs $(15-18,20,22)$ with 398 patients examined the effect of omega- 3 fatty acids supplementation on serum glucose. We used the random effects model for analysis due to the high heterogeneity in the studies $\left(\mathrm{P}=0.001, \mathrm{I}^{2}=73 \%\right)$, and the results indicated that omega-3 fatty acids supplementation, compared with the control group, did not change serum glucose in PCOS patients (Figure 3: $-0.76 \mathrm{mg} / \mathrm{dL}, 95 \% \mathrm{CI}:-1.71$ to $0.19 \mathrm{mg} / \mathrm{dL}$, $\mathrm{P}=0.12)$.

A total of 6 studies $(15-17,19,20,22)$ involving 506 participants reported the effect of omega- 3 fatty acids supplementation on the serum insulin in PCOS patients. No heterogeneity was found among these studies $(\mathrm{P}=0.60$,
$\mathrm{I}^{2}=0$ ). Thus, we selected the fixed effects model for analysis and the results demonstrated that compared with the control group, the supplementation of omega-3 fatty acid could significantly reduce serum insulin (Figure 4: $-2.58 \mathrm{pmol} / \mathrm{L}, 95 \% \mathrm{CI}:-3.34$ to $-1.82 \mathrm{pmol} / \mathrm{L}, \mathrm{P}<0.00001$ ).

Data on the effect of the therapy with omega-3 fatty acids on HOMA-IR were reported in 6 RCTs $(15-17,19,20,22)$ involving 506 patients. We used the fixed effects model for analysis due to the low heterogeneity among the studies $\left(\mathrm{P}=0.41, \mathrm{I}^{2}=2 \%\right)$. Compared with the control group, the omega-3 fatty acid supplementation group had bigger decreases in HOMA-IR in patients with PCOS (Figure 5: $-0.57,95 \%$ CI: -0.75 to $-0.40, \mathrm{P}<0.00001)$.

\section{Effect of omega-3 fatty acids supplementation on serum TC, serum triglyceride, serum LDL-C, serum VLDL-C, serum HDL-C, and hs-CRP}

A total of 8 studies $(15,17-23)$ involving 654 participants reported the effect of omega-3 fatty acids supplementation on TC, with statistical heterogeneity among the studies $\left(\mathrm{P}<0.00001, \mathrm{I}^{2}=95 \%\right)$. Thus, the random effects model was used for analysis and the results showed that compared with the control group, omega-3 fatty acids supplementation statistically reduced TC in patients with PCOS (Figure 6: $-6.87 \mathrm{mg} / \mathrm{dL}, 95 \% \mathrm{CI}:-10.28$ to $-3.47 \mathrm{mg} / \mathrm{dL}, \mathrm{P}<0.0001)$.

The effect of omega-3 fatty acids supplementation on serum triglyceride was evaluated in 8 RCTs $(15,17-23)$ with 654 patients. We used the random effects model for analysis due to the high statistical heterogeneity among the studies $\left(\mathrm{P}<0.00001, \mathrm{I}^{2}=97 \%\right)$. The results indicated that compared with the control group, omega-3 fatty acids supplementation statistically lowered serum triglyceride in patients with PCOS (Figure 7: $-4.03 \mathrm{mg} / \mathrm{dL}, 95 \%$ CI: -5.53 to $-2.52 \mathrm{mg} / \mathrm{dL}, \mathrm{P}<0.00001)$.

The meta-analysis included 6 studies $(15,17,19-21,23)$ involving 525 participants which reported the effects of omega-3 fatty acids supplementation on serum LDL-C. It was demonstrated that omega- 3 fatty acids supplementation statistically reduced LDL-C (Figure 8: $-6.64 \mathrm{mg} / \mathrm{dL}, 95 \%$ CI: -11.58 to $-1.70 \mathrm{mg} / \mathrm{dL}, \mathrm{P}=0.008)$. A high statistical heterogeneity was detected among the studies $(\mathrm{P}<0.00001$, $\left.\mathrm{I}^{2}=99 \%\right)$, and the random effects model was used.

Only 3 RCTs $(15,20,23)$ with 182 patients reported the effect of omega-3 fatty acids supplementation on VLDL-C in PCOS patients. The random effect model was used due to the high statistical heterogeneity among the studies $\left(\mathrm{P}=0.03, \mathrm{I}^{2}=72 \%\right)$. The results showed that omega- 3 fatty acids supplementation statistically lowered serum VLDL-C 


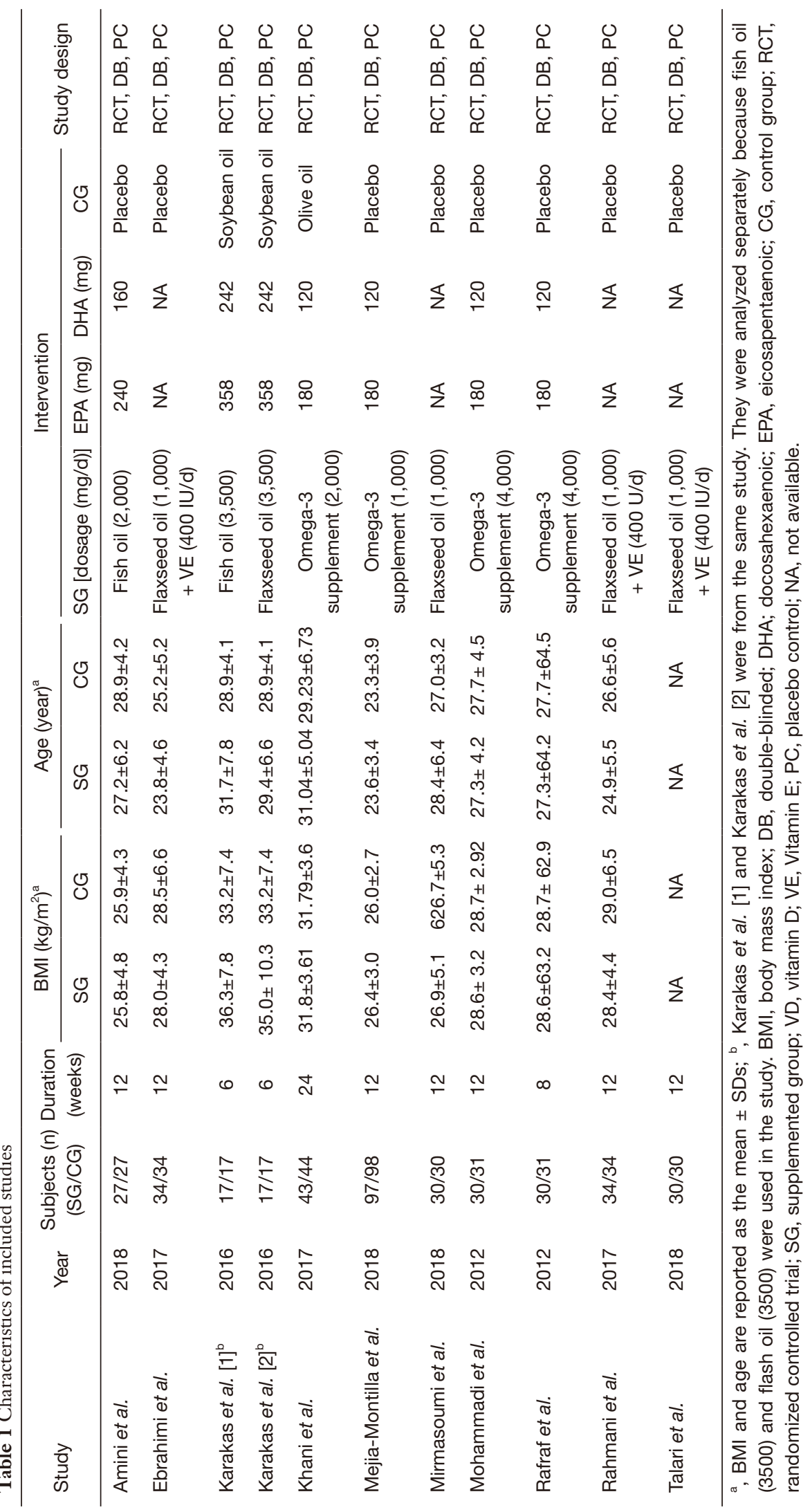


A

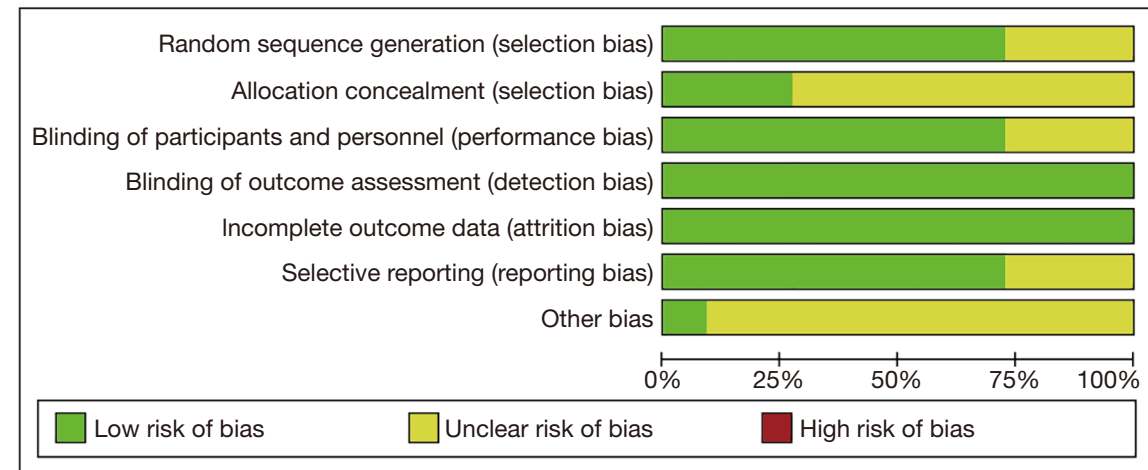

B

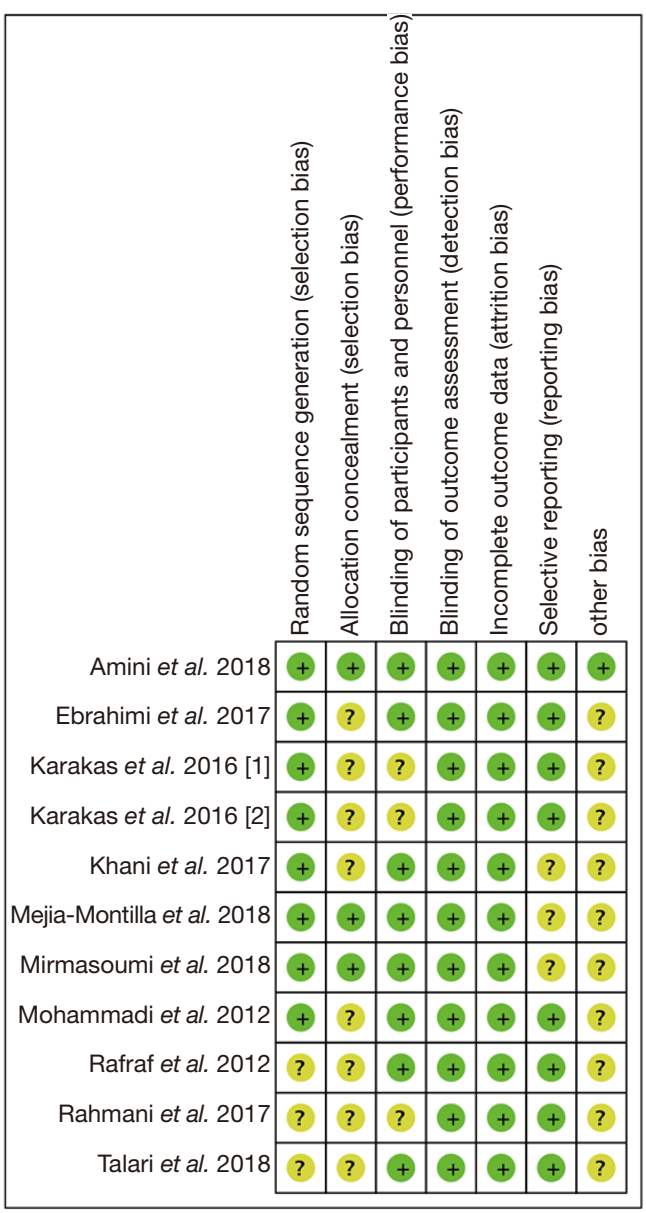

Figure 2 The Cochrane bias risk assessment tool was used to assess the bias risk, and the quality of included studies was assessed (A) bias risk summary (B) overall assessment of bias risk.

in patients with PCOS (Figure 9: $-3.29 \mathrm{mg} / \mathrm{dL}, 95 \% \mathrm{CI}$ : -6.54 to $-0.05 \mathrm{mg} / \mathrm{dL}, \mathrm{P}=0.05)$.

A total of 7 studies $(15,17-21,23)$ with 593 subjects had data on the effect of omega-3 fatty acids supplementation on serum HDL-C. The overall results demonstrated that omega-3 fatty acids supplementation statistically improved serum HDL-C in patients with PCOS (Figure 10: $2.94 \mathrm{mg} / \mathrm{dL}$, $95 \%$ CI: 0.63 to $5.26 \mathrm{mg} / \mathrm{dL}, \mathrm{P}=0.01)$. We used the random effects model for analysis on account of the high statistical heterogeneity among the studies $\left(\mathrm{P}<0.00001, \mathrm{I}^{2}=87 \%\right)$. 


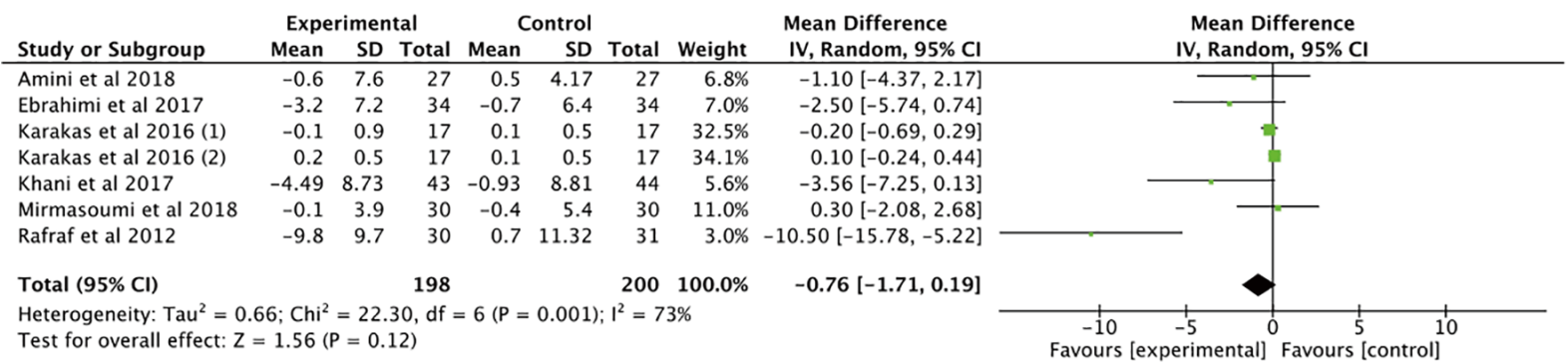

Figure 3 Results of the effect of omega-3 fatty acids supplementation on glucose.

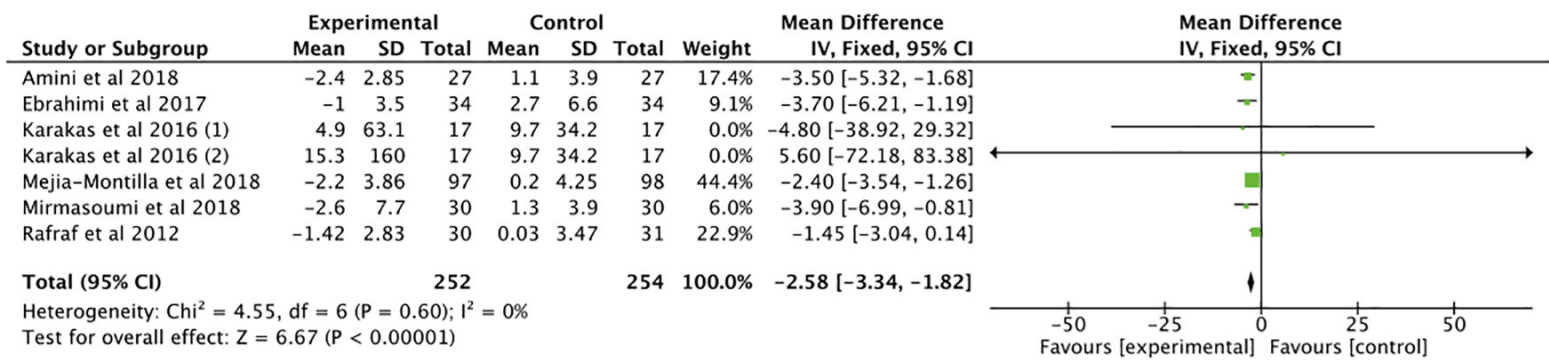

Figure 4 Results of the effect of omega-3 fatty acids supplementation on insulin.

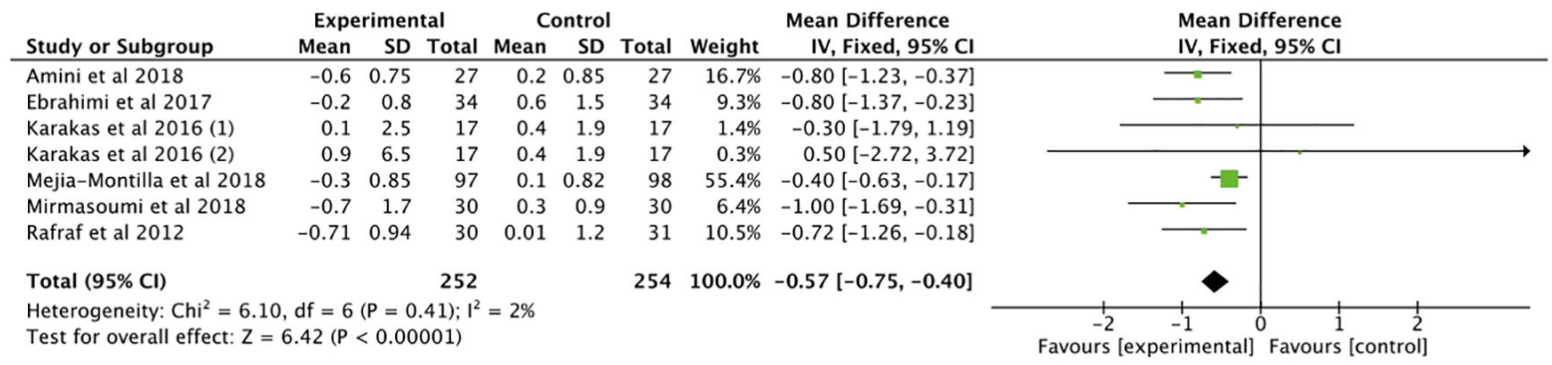

Figure 5 Results of the effect of omega-3 fatty acids supplementation on HOMA-IR. HOMA-IR, homeostatic model assessment-insulin resistance.

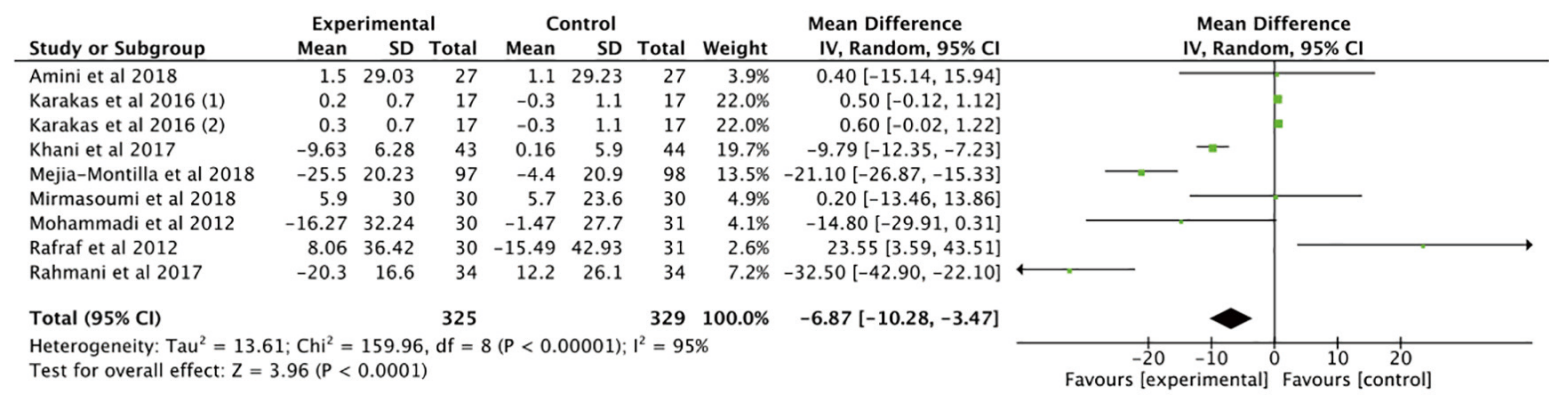

Figure 6 Results of the effect of omega-3 fatty acids supplementation on TC. TC, total cholesterol. 


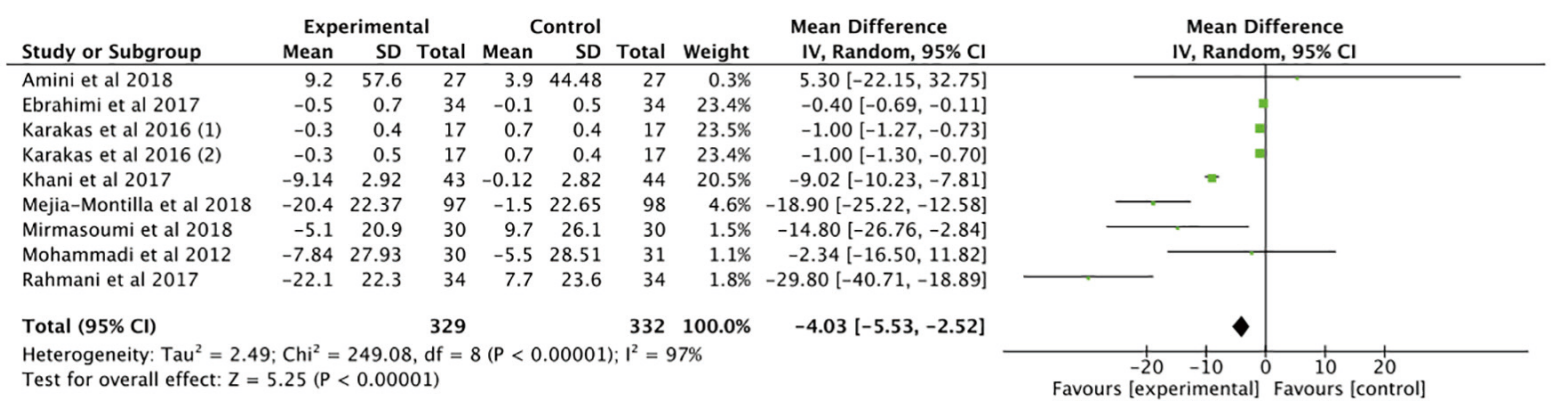

Figure 7 Results of the effect of omega-3 fatty acids supplementation on triglyceride.

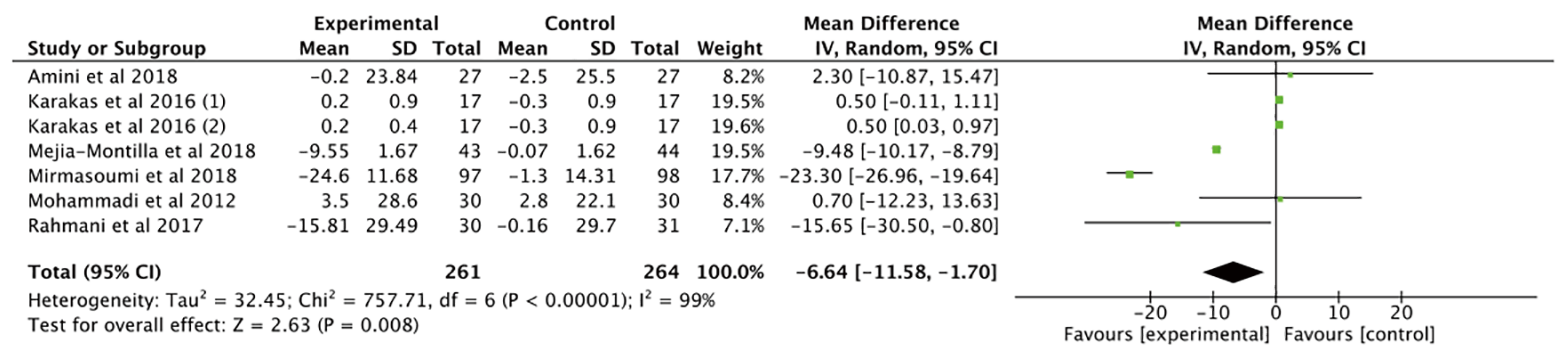

Figure 8 Results of the effect of omega-3 fatty acids supplementation on LDL-C. LDL-C, serum low-density lipoprotein cholesterol.

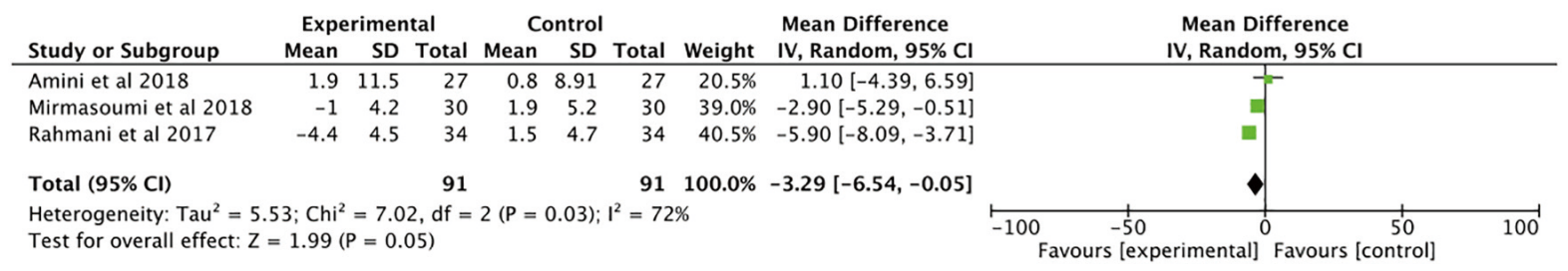

Figure 9 Results of the effect of omega-3 fatty acids supplementation on VLDL-C. VLDL-C, serum very low-density lipoprotein cholesterol.

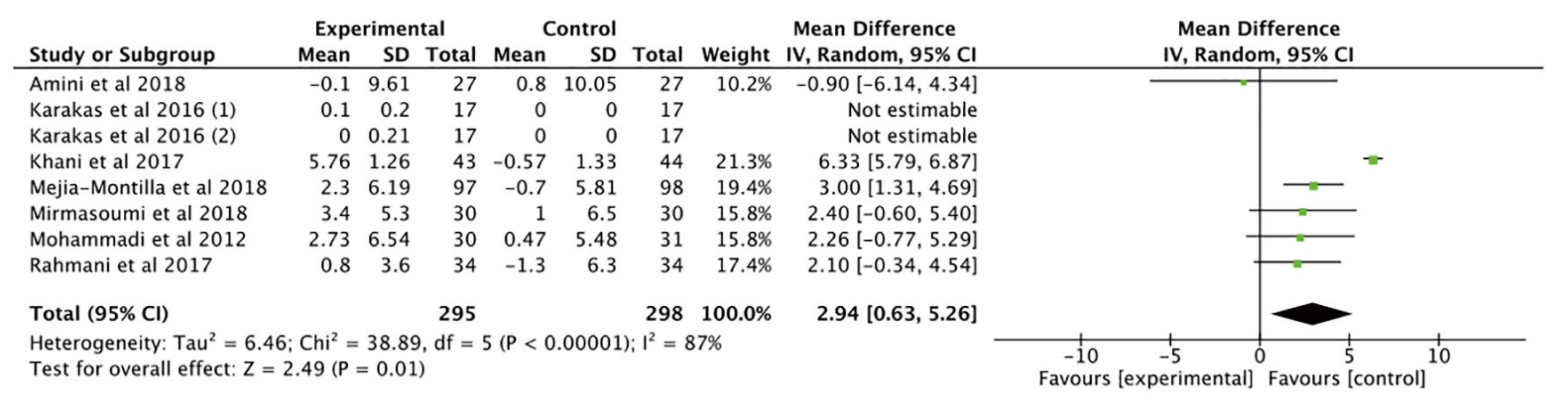

Figure 10 Results of the effect of omega-3 fatty acids supplementation on HDL-C. HDL-C, serum high-density lipoprotein cholesterol. 


\begin{tabular}{|c|c|c|c|c|c|c|c|c|c|c|c|}
\hline \multirow[b]{2}{*}{ Study or Subgroup } & \multicolumn{3}{|c|}{ Experimental } & \multicolumn{3}{|c|}{ Control } & \multicolumn{2}{|r|}{ Mean Difference } & \multirow{2}{*}{\multicolumn{2}{|c|}{$\begin{array}{c}\text { Mean Difference } \\
\text { IV, Random, } 95 \% \mathrm{CI}\end{array}$}} & \\
\hline & Mean & SD & Total & Mean & SD & Total & Weight & IV, Random, $95 \% \mathrm{CI}$ & & & \\
\hline Karakas et al 2016 (1) & -0.4 & 2.4 & 17 & 0.1 & 1.2 & 17 & $32.9 \%$ & $-0.50[-1.78,0.78]$ & - & & \\
\hline Karakas et al 2016 (2) & -0.1 & 3.2 & 17 & 0.1 & 1.2 & 17 & $32.8 \%$ & $-0.20[-1.82,1.42]$ & -1 & - & \\
\hline Mirmasoumi et al 2018 & -1.6 & 3.1 & 30 & 0.2 & 1.5 & 30 & $32.9 \%$ & $-1.80[-3.03,-0.57]$ & -- & & \\
\hline Talari et al 2018 & -360.2 & 140.1 & 30 & 206.6 & 140.1 & 30 & $1.4 \%$ & $-566.80[-637.70,-495.90]$ & 1 & & \\
\hline Total $(95 \% \mathrm{Cl})$ & & & 94 & & & 94 & $100.0 \%$ & $-8.97[-17.66,-0.28]$ & & & \\
\hline $\begin{array}{l}\text { Heterogeneity: } \mathrm{Tau}^{2}=5 \\
\text { Test for overall effect: } \mathrm{Z}\end{array}$ & $\begin{array}{l}.32 ; \mathrm{Chi}^{2} \\
=2.02(\mathrm{P}=\end{array}$ & $\begin{array}{l}=247.7 \\
=0.04)\end{array}$ & $79, \mathrm{df}=$ & $=3(P<$ & 0.0000 & 1); $1^{2}=$ & $=99 \%$ & & $\begin{array}{ccc}-10 & -5 & 1 \\
\text { Favours [experimental] }\end{array}$ & $\begin{array}{l}1 \\
0\end{array}$ & $\begin{array}{l}10 \\
10\end{array}$ \\
\hline
\end{tabular}

Figure 11 Results of the effect of omega-3 fatty acids supplementation on hs-CRP. hs-CRP, high-sensitivity C-reactive protein.

The meta-analysis of 3 RCTs $(17,20,24)$ with 188 patients showed that omega-3 fatty acids supplementation statistically changed the serum hs-CRP in patients with PCOS (Figure 11: $-8.97 \mathrm{mg} / \mathrm{dL}, 95 \% \mathrm{CI}:-17.66$ to $-0.28 \mathrm{mg} / \mathrm{dL}, \mathrm{P}=0.04$ ). There was a high statistical heterogeneity among the studies $\left(\mathrm{P}<0.00001, \mathrm{I}^{2}=99 \%\right)$ and therefore the random effects model analysis was used.

\section{Heterogeneity analysis}

There was some heterogeneity in the findings of our study, and we speculate that the heterogeneity may have come from different omega-3 fatty acid supplementation, treatment process, intervention type, and frequency. In addition, differences in age, patient health status (basal omega-3 fatty acid level, comorbidity rate, and so on) and study quality may have been additional sources of heterogeneity.

\section{Publication bias}

We evaluated the publication bias of the outcome indicators (glucose, insulin, HOMA-IR, triglyceride, TC, LDL-C, VLDL-C, HDL-C, and hs-CRP) with a funnel plot, and the results are shown in Figures 12 and 13.

\section{Discussion}

The disease PCOS is becoming a major health problem due to its high prevalence and high risk of cardiovascular disease. Supplementation of omega-3 fatty acids as a potential treatment for PCOS has attracted wide attention. In present systematic review and meta-analysis, we systematically reviewed 10 available RCTs with 778 patients evaluating the efficacy of omega- 3 fatty acids supplementation on cardiovascular risks factors in PCOS patients. The pooled results revealed that compared with placebo, omega- 3 fatty acids supplementation had significant reduction effects on serum insulin, HOMA-IR, TC, triglyceride, LDL-C,
VLDL-C, HDL-C, and hs-CRP among PCOS patients, while changes in serum glucose were not significant.

The condition of PCOS is clinically heterogeneous, in part due to genetic and environmental factors (25). In addition to reproductive consequences including hyperandrogenism, menstrual cycle disturbances, and infertility, PCOS is also characterized by the presence of cardiovascular comorbidities, such as IR, dyslipidemia, obesity, and hypertension $(4,26)$.

The presence of IR with consequent increased likelihood of further developing type II diabetes mellitus (T2D) is indeed a common finding in PCOS, with a prevalence of $50-70 \%(3,27)$. Research has shown that insulin has a gonadotropin-like action and modulates ovarian steroidogenesis; insulin signaling disruption in the brain can effect ovulation and body weight regulation (28). Meanwhile, hyperinsulinemia favors hypertension through endothelin-1, and dyslipidemia (29). Dyslipidemia is certainly highly prevalent symptom of PCOS. Based on the NCEP guidelines, up to $70 \%$ of women with PCOS have dyslipidemia (3), which may increase the risk of atherosclerosis and CVD in patients with PCOS (5). The marker hs-CRP, among the wide array of inflammatory biomarkers, has received the most attention for its use in the propagation of atherosclerosis and susceptibility to cardiovascular events (30).

In addition to lifestyle modifications and weight loss, nutrition intervention is also a successful treatment for PCOS, among which, omega-3 fatty acids have attracted a lot of interest due to the beneficial effect on cardiometabolic risk factors in PCOS. Previous studies have shown that a greater plasma LC omega- 3 fatty acids status was associated with a less atherogenic lipid profile (5). A study by Fakhrzadeh et al. indicated that supplementation with low dose omega-3 fatty acids for 6 months has a meaningful effect on the reduction of serum triglyceride levels in the elderly (31). In our meta-analysis, the pooled results indicated that PCOS patients treated with vitamin 

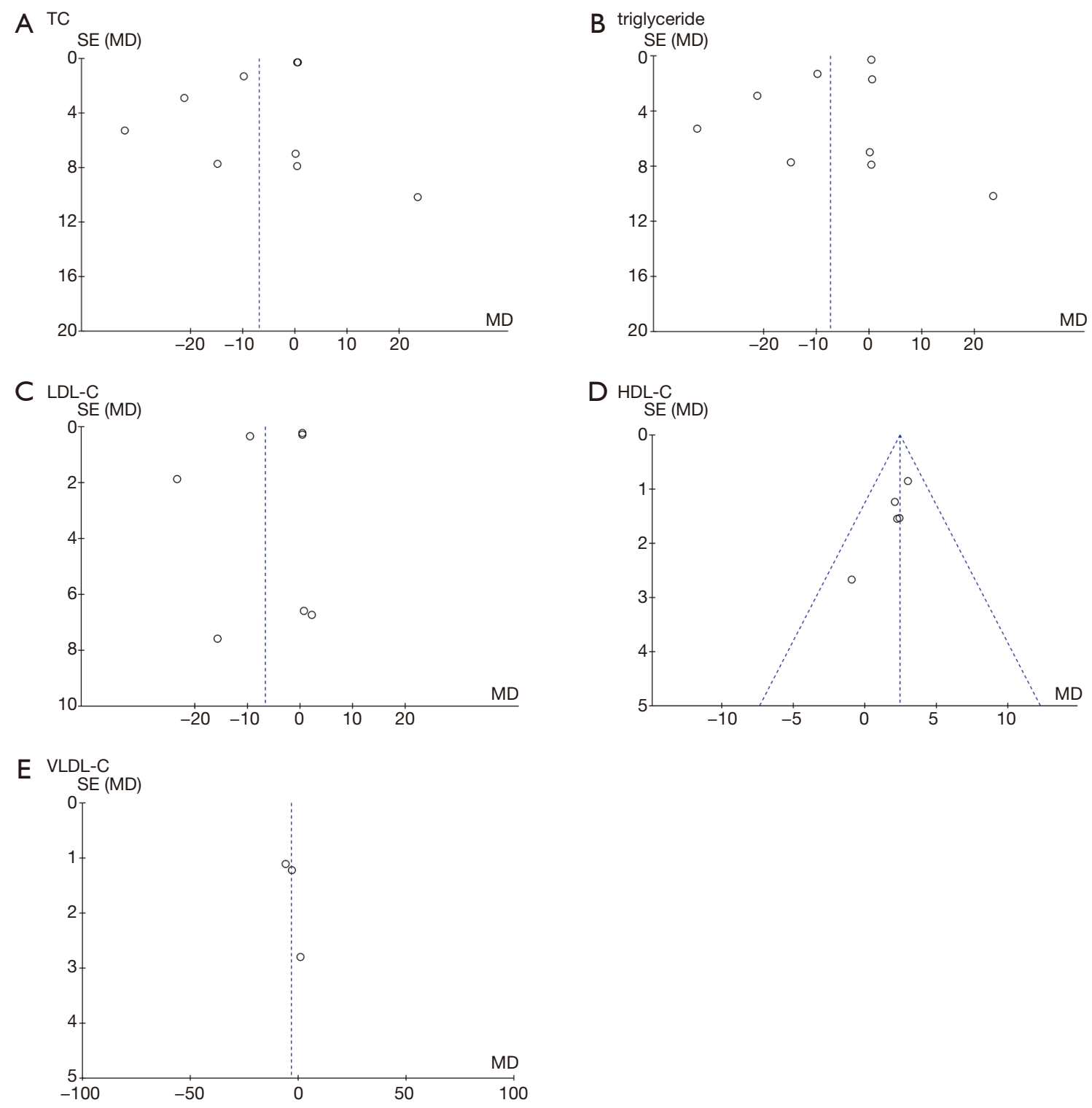

Figure 12 Funnel plot of SE by for (A) TC, (B) triglyceride, (C) LDL-C, (D) HDL-C, (E) VLDL-C, detailing publication bias in the studies selected for analysis. Closed circles represent observed published studies. SE, standard error; SMD, standardized mean difference; TC, total cholesterol; LDL-C, serum low-density lipoprotein cholesterol; HDL-C, serum high-density lipoprotein cholesterol; VLDL-C, serum very low-density lipoprotein cholesterol.

D had associated improvements in insulin, HOMA-IR, TC, triglyceride, LDL-C, VLDL-C, HDL-C, hs-CRP levels, but no effect on glucose was observed. The exact mechanism by which omega-3 fatty acids supplementation affects cardiovascular risk factors have not yet been elucidated. It has been demonstrated that omega-3 fatty acids supplementation may improve IR via producing and secreting anti-inflammatory adipokine and also by reducing inflammation and proinflammatory cytokines. Besides, Gray et al. pointed out that omega-3 fatty acids play a beneficial role through the modulation of the amount and type of eicosapentaenoic acid, as well as regulating intracellular signaling pathways and transcription factor activities (32).

This systematic review and meta-analysis had some limitations. Firstly, the studies included had relatively small numbers of participants. Secondly, there was a high degree 

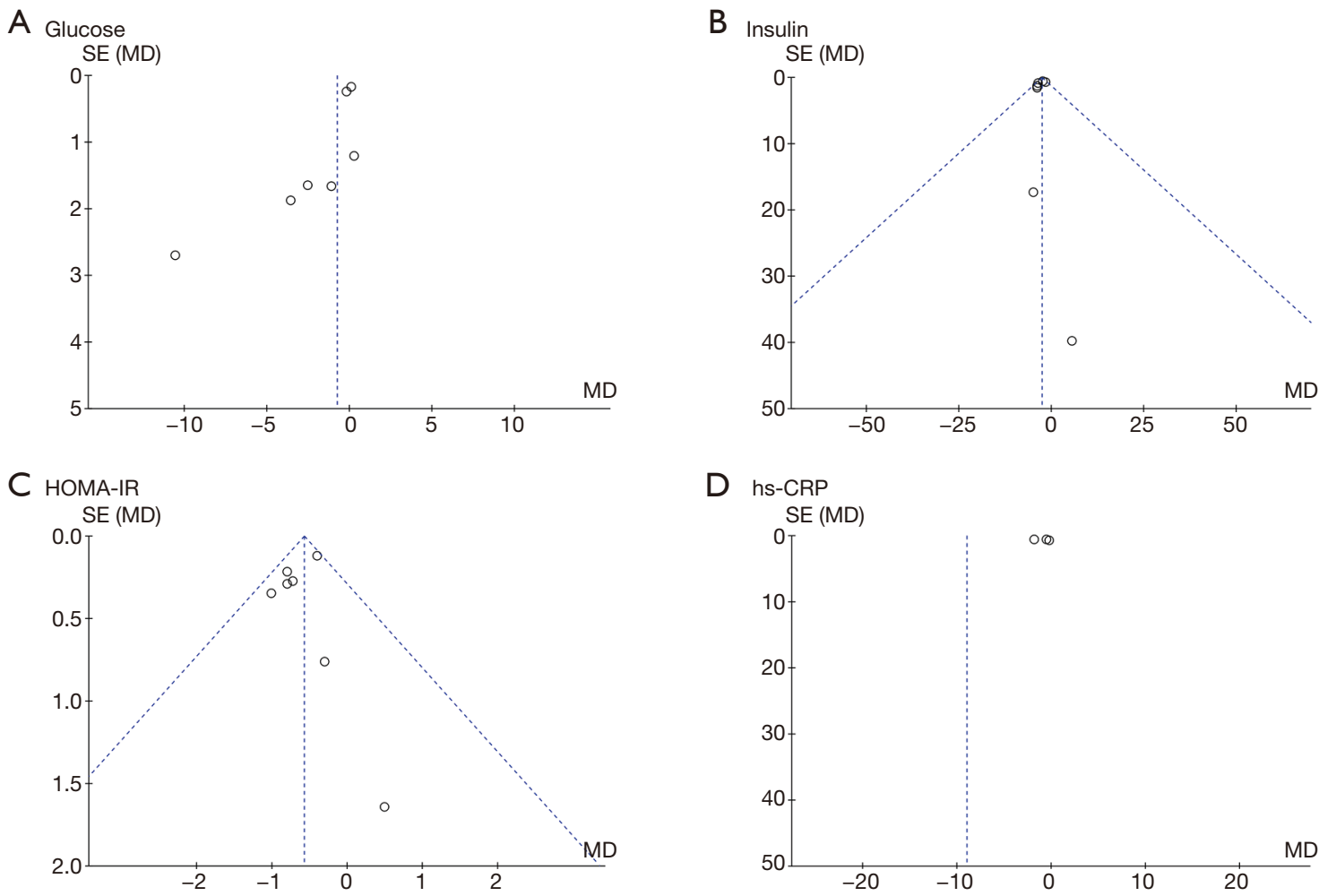

Figure 13 Funnel plot of SE by standardized mean difference for (A) glucose, (B) insulin, (C) HOMA-IR, (D) hs-CRP, detailing publication bias in the studies selected for analysis. Closed circles represent observed published studies. SE, standard error; SMD, standardized mean difference; HOMA-IR, homeostatic model assessment-insulin resistance; hs-CRP, high-sensitivity C-reactive protein.

of heterogeneity between the studies, which may have led to a high risk of bias. Thirdly, the participants might have had different status of PCOS. Finally, due to the small number of included studies and samples, we did not conduct subgroup analysis.

\section{Conclusions}

So far, evidence from RCTs has shown that compared with placebo, omega-3 fatty acids supplementation has significant reduced serum insulin, HOMA-IR, TC, triglyceride, LDL-C, VLDL-C, HDL-C, and hs-CRP in patients with PCOS, while changes in glucose were not significant. In addition, considering the high prevalence of disorders of glucose and lipid metabolism in PCOS patients, it is suggested that omega- 3 supplementation in the population may be a simple and low-risk additional treatment for women with PCOS. However, due to the limitations of our study, the effect of omega-3 supplementation on cardiovascular risk in PCOS patients should be investigated further through large-scale RCTs, including appropriate dose, duration, and type of supplementation.

\section{Acknowledgments}

Funding: None.

\section{Footnote}

Reporting Checklist: The authors have completed the PRISMA reporting checklist. Available at https://dx.doi. org/10.21037/apm-21-1050

Conflicts of Interest: All authors have completed the ICMJE uniform disclosure form (available at https://dx.doi. org/10.21037/apm-21-1050). The authors have no conflicts of interest to declare.

Ethical Statement: The authors are accountable for all aspects of the work in ensuring that questions related 
to the accuracy or integrity of any part of the work are appropriately investigated and resolved.

Open Access Statement: This is an Open Access article distributed in accordance with the Creative Commons Attribution-NonCommercial-NoDerivs 4.0 International License (CC BY-NC-ND 4.0), which permits the noncommercial replication and distribution of the article with the strict proviso that no changes or edits are made and the original work is properly cited (including links to both the formal publication through the relevant DOI and the license). See: https://creativecommons.org/licenses/by-nc-nd/4.0/.

\section{References}

1. Wekker V, van Dammen L, Koning A, et al. Long-term cardiometabolic disease risk in women with PCOS: a systematic review and meta-analysis. Hum Reprod Update 2020;26:942-60.

2. Dokras A. Cardiovascular disease risk in women with PCOS. Steroids 2013;78:773-6.

3. Legro RS, Kunselman AR, Dunaif A. Prevalence and predictors of dyslipidemia in women with polycystic ovary syndrome. Am J Med 2001;111:607-13.

4. Escobar-Morreale HF. Polycystic ovary syndrome: definition, aetiology, diagnosis and treatment. Nat Rev Endocrinol 2018;14:270-84.

5. Osibogun O, Ogunmoroti O, Michos ED. Polycystic ovary syndrome and cardiometabolic risk: Opportunities for cardiovascular disease prevention. Trends Cardiovasc Med 2020;30:399-404.

6. Phelan N, O'Connor A, Kyaw Tun T, et al. Hormonal and metabolic effects of polyunsaturated fatty acids in young women with polycystic ovary syndrome: results from a cross-sectional analysis and a randomized, placebo-controlled, crossover trial. Am J Clin Nutr 2011;93:652-62.

7. Santos HO, Price JC, Bueno AA. Beyond Fish Oil Supplementation: The Effects of Alternative Plant Sources of Omega-3 Polyunsaturated Fatty Acids upon Lipid Indexes and Cardiometabolic Biomarkers-An Overview. Nutrients 2020;12:3159.

8. Oner G, Muderris, II. Efficacy of omega-3 in the treatment of polycystic ovary syndrome. J Obstet Gynaecol 2013;33:289-91.

9. Kabir M, Skurnik G, Naour N, et al. Treatment for 2 mo with $n 3$ polyunsaturated fatty acids reduces adiposity and some atherogenic factors but does not improve insulin sensitivity in women with type 2 diabetes: a randomized controlled study. Am J Clin Nutr 2007;86:1670-9.

10. Akinkuolie AO, Ngwa JS, Meigs JB, et al. Omega-3 polyunsaturated fatty acid and insulin sensitivity: a metaanalysis of randomized controlled trials. Clin Nutr 2011;30:702-7.

11. Shamseer L, Moher D, Clarke M, et al. Preferred reporting items for systematic review and meta-analysis protocols (PRISMA-P) 2015: elaboration and explanation. BMJ 2015;350:g7647.

12. Jørgensen L, Paludan-Muller AS, Laursen DR, et al. Evaluation of the Cochrane tool for assessing risk of bias in randomized clinical trials: overview of published comments and analysis of user practice in Cochrane and non-Cochrane reviews. Syst Rev 2016;5:80.

13. Wan X, Wang W, Liu J, et al. Estimating the sample mean and standard deviation from the sample size, median, range and/or interquartile range. BMC Med Res Methodol 2014;14:135.

14. Duval S, Tweedie R. Trim and fill: A simple funnel-plotbased method of testing and adjusting for publication bias in meta-analysis. Biometrics 2000;56:455-63.

15. Amini M, Bahmani F, Foroozanfard F, et al. The effects of fish oil omega-3 fatty acid supplementation on mental health parameters and metabolic status of patients with polycystic ovary syndrome: a randomized, double-blind, placebo-controlled trial. J Psychosom Obstet Gynaecol 2018. doi: 10.1080/0167482X.2018.1508282.

16. Ebrahimi FA, Samimi M, Foroozanfard F, et al. The Effects of Omega-3 Fatty Acids and Vitamin E CoSupplementation on Indices of Insulin Resistance and Hormonal Parameters in Patients with Polycystic Ovary Syndrome: A Randomized, Double-Blind, PlaceboControlled Trial. Exp Clin Endocrinol Diabetes 2017;125:353-9.

17. Karakas SE, Perroud B, Kind T, et al. Changes in plasma metabolites and glucose homeostasis during omega-3 polyunsaturated fatty acid supplementation in women with polycystic ovary syndrome. BBA Clin 2016;5:179-85.

18. Khani B, Mardanian F, Fesharaki SJ. Omega-3 supplementation effects on polycystic ovary syndrome symptoms and metabolic syndrome. J Res Med Sci 2017;22:64.

19. Mejia-Montilla J, Reyna-Villasmil E, Domínguez-Brito L, et al. Supplementation with omega-3 fatty acids and plasma adiponectin in women with polycystic ovary syndrome. Endocrinol Diabetes Nutr (Engl Ed) 2018;65:192-9. 
20. Mirmasoumi G, Fazilati M, Foroozanfard F, et al. The Effects of Flaxseed Oil Omega-3 Fatty Acids Supplementation on Metabolic Status of Patients with Polycystic Ovary Syndrome: A Randomized, DoubleBlind, Placebo-Controlled Trial. Exp Clin Endocrinol Diabetes 2018;126:222-8.

21. Mohammadi E, Rafraf M. Benefits of omega-3 Fatty acids supplementation on serum paraoxonase 1 activity and lipids ratios in polycystic ovary syndrome. Health Promot Perspect 2012;2:197-204.

22. Rafraf M, Mohammadi E, Asghari-Jafarabadi M, et al. Omega-3 fatty acids improve glucose metabolism without effects on obesity values and serum visfatin levels in women with polycystic ovary syndrome. J Am Coll Nutr 2012;31:361-8.

23. Rahmani E, Samimi M, Ebrahimi FA, et al. The effects of omega-3 fatty acids and vitamin E co-supplementation on gene expression of lipoprotein(a) and oxidized low-density lipoprotein, lipid profiles and biomarkers of oxidative stress in patients with polycystic ovary syndrome. Mol Cell Endocrinol 2017;439:247-55.

24. Talari HR, Poladchang S, Hamidian Y, et al. The Effects of Omega-3 and Vitamin E Co-supplementation on Carotid Intima-media Thickness and Inflammatory Factors in Patients with Polycystic Ovary Syndrome. Oman Med J 2018;33:473-9.

25. Lorenz LB, Wild RA. Polycystic ovarian syndrome: an evidence-based approach to evaluation and management of diabetes and cardiovascular risks for today's clinician. Clin Obstet Gynecol 2007;50:226-43.

Cite this article as: Xia Y, Wang Y, Cui M, Su D. Efficacy of omega-3 fatty acid supplementation on cardiovascular risk factors in patients with polycystic ovary syndrome: a systematic review and meta-analysis. Ann Palliat Med 2021;10(6):64256437. doi: 10.21037/apm-21-1050
26. McCartney CR, Marshall JC. CLINICAL PRACTICE. Polycystic Ovary Syndrome. N Engl J Med 2016;375:54-64.

27. Kakoly NS, Earnest A, Teede HJ, et al. The Impact of Obesity on the Incidence of Type 2 Diabetes Among Women With Polycystic Ovary Syndrome. Diabetes Care 2019;42:560-7.

28. Diamanti-Kandarakis E, Dunaif A. Insulin resistance and the polycystic ovary syndrome revisited: an update on mechanisms and implications. Endocr Rev 2012;33:981-1030.

29. Diamanti-Kandarakis E, Paterakis T, Alexandraki K, et al. Indices of low-grade chronic inflammation in polycystic ovary syndrome and the beneficial effect of metformin. Hum Reprod 2006;21:1426-31.

30. Yousuf O, Mohanty BD, Martin SS, et al. Highsensitivity $\mathrm{C}$-reactive protein and cardiovascular disease: a resolute belief or an elusive link? J Am Coll Cardiol 2013;62:397-408.

31. Fakhrzadeh H, Ghaderpanahi M, Sharifi F, et al. The effects of low dose n-3 fatty acids on serum lipid profiles and insulin resistance of the elderly: a randomized controlled clinical trial. Int J Vitam Nutr Res 2010;80:107-16.

32. Gray B, Steyn F, Davies PSW, et al. Omega-3 fatty acids: a review of the effects on adiponectin and leptin and potential implications for obesity management. Eur J Clin Nutr 2013;67:1234-42.

(English Language Editor: J. Jones) 